Article

\title{
Middle ear packing and external ear packing with fibrin glue enhances the success of fat myringoplasty without hyaluronic acid disc
}

\author{
Jongmin Kim ${ }^{1}$ and Chul Ho Jang ${ }^{2, *}$ \\ Department of Otolaryngology, Chonnam National University Hospital1; huberjoads@naver.com \\ Correspondence: chulsavio@hanmail.net; Tel.: +82 622206774
}

\begin{abstract}
Background: To date, FM using middle ear packing has rarely reported. In this study, we hypothesized that middle ear packing with dexamethasone soaked gelfoam and fibrin glue over the fat graft could promote the success rate of TMP closure regardless of perforation size. Methods: Between January 2005 and July 2020, a total of 209 patients who underwent fat myringoplasty due to chronic TMP at tertiary referral center were encountered and analyzed. The success rate and audiologic outcome was analyzed. Results: The mean successful TM closure rate was $88.0 \%$. The success rate by different age generation was not significant difference. The size of the perforation grade showed no significant difference. The perforation site by anterior or posterior was not significant. The preoperative mean threshold of AC, BC and ABG were 55.71, 12.98 and $42.73 \mathrm{~dB}$ respectively. The postoperative mean threshold of $\mathrm{AC}, \mathrm{BC}$ and $\mathrm{ABG}$ were 23.67, 12.98 and $10.69 \mathrm{~dB}$. The change of preoperative and postoperative hearing showed statistically significant. Conclusion: From our results, the simple trimming of the perforation edge and middle ear packing with external ear canal packing using fibrin glue induced the stable adhesion between the remnant TM and the fat graft.
\end{abstract}

Keywords: tympanic membrane; chronic perforation; fat myringoplasty; success rate; middle ear packing;fibrin glue packing

\section{Introduction}

A myringoplasty which is standard care of chronic tympanic membrane perforation (TMP) is commonly performed in otologic field, due to the high incidence of tympanic membrane perforation. To perform tympanoplasty for the repair of TMP, various autologous materials such as temporalis fascia, perichondrium, or cartilage graft have been used depending on the surgeon's choice. As for graft, temporalis fascia is the most common material used for the closure of tympanic membrane perforation.

Fat myringoplasty appears safe and suitable for stable, dry, small sized perforations or geriatric patients with underlying disease. Since Ringerberg [1] introduced for the first time, it has been accepted successful technique for small TMP and geriatric patients [2-4]. According to the publicated reports, average success rate of fat myringoplasty has been reported from $72.9 \%$ to $92.2 \%$ [2,5-10]. Recently, Salabi et al introduced that the fat myringoplasty with hyaluronic acid (Epidisc Otologic Lamina; Xomed-Medtronic, Jacksonville, FL) enhanced the success rate compared to simple fat myringoplasty as well small perforation as large perforation of the tympanic membrane [11-16]. The theory in this study was to provide a more natural scaffold using hyaluronic acid for keratinocyte migration. The classical fat myringoplasty technique consists of de-epithelialization of the perforation edges and subsequently filling the perforation using fat tissue from earlobe or abdominal fat.

Saliba recommended the lateral fat bulging should not be too high, middle ear cavity should be filled with two third of the fat and one-third fat is lateral fat bulging in the external ear canal [17]. When middle ear cavity is filled with large fat, the fat is easy to contact the ossicle and fat fat may obliterate the middle ear space which induce poor acoustic properties. 
In our experience, external fat over the perforation, it will change as flat as time goes by, eventually same level of the tympanic membrane, however, fate of the fat in the middle ear is difficult to assess by otoendoscopy. Histopathological study of human revealed that fate of the fat in the middle ear after fat myringoplasty, fat preserved its volume covered by fibrous layer [18].. If the fat contacted to the middle ear mucosa, it will be remained as focal adhesion. If the site is near to the protympanum, it can be cause of obstruction of the Eustachian tube.

In this study, we have used middle ear gelfoam packing before fat filling for the stability and prevent to adhesion of the fat to the promontory or protympanum using dexamethasone soaked gelfoam.

Gelfoam is frequently used as a middle ear packing material in the tympanoplasty. The majority of the gelfoam can be resorbed as time goes by. However, resorption of the gelfoam does not work well in case of eustachian tube dysfunction or presence of mucosal defect, finally it can frequently induce middle ear fibrosis or adhesions [19]. Previous our experimental study has reported that gelfoam soaked with a corticosteroid prevent the fibrosis in the middle ear [20].. Experimentally and clinically, topical or dexamethasone injection during surgery has been shown to be protective for the inner ear [21,22].

To date, fat myringoplasty using middle ear packing with antibiotic-dexamethasone coated gelfoam has not been reported. Previously we experienced middle ear packing by antibiotic-dexamethasone soaked gelfoam in the geriatric patient[23]. It showed good result.

The purpose of the present study was to investigate the success rate fat myringoplasty with middle ear packing using gelfoam instead of simple fat filling. Because this middle ear packing supports even distribution of fat tissue easily contact the entire perforation margin.

\section{Materials and Methods}

In a retrospective chart review of fat myringoplasty, performed at our tertiary otolaryngologic care centers between January 2005 and July 2020, a total of 209 patients with chronic otitis media were encountered. This study was approved by internal review of board (approval No. CNUH-2020-153). The duration for the perforation was more than 12 moths, and the patients were referred to senior surgeon for fat myringoplsaty. The perforation was captured by otoendoscopy $\left(2.7 \mathrm{~mm}, 0^{\circ}\right.$ Karl Storz Co. Tuttlingen, German) and saved in the electronic chart. Pure tone audiometry and temporal bone $\mathrm{CT}$ were routinely performed. Eustachian tube function was indirectly assessed by pneumatization of the mastoid air cell in temporal bone CT. Active otorrhea, cholesteatoma, marginal perforation, and ossicular defect were excluded.

The perforation size was divided 4 groups according to the modified Saliba's subdivision [17]. The pinhole perforation was considered less than $10 \%$. The four groups by the perforation size by the 4 quadrants of the tympanic membrane. Pinhole perforation, small perforation (grade I, less than 25\%), medium perforation (grade II, 25-50\%), and large perforation (over than $50 \%$ ).

\section{Surgical procedure}

All fat myringoplastes were under local anesthesia using infiltration of dental lidocaine (1\% lidocaine with 1 : 100000 epinephrine) with transcanal approach by surgical microscope. After antiseptic painting, incision was made along the earlobe margin. After fat was taken from earlobe, incision was approximated by nylon. Using ear speculum, de-epithelialization of the perforation rim prior to repair was performed by sharp pick and alligator cup forcep. After middle ear and external ear canal irrigation using normal saline, the middle ear packing was performed using ciprofloxacin-dexamethasone soaked gelfoam according to the size of the perforation. If the perforation was small, the compressed gelfoam which was cut in the shape of toothpick was inserted into the middle ear throught the 
perforation especially in the protympanum because fat may intrude into the protympanum (Fig.1). And the ciprofloxacin-dexamethasone was dropped over the gelfoam by $1 \mathrm{ml}$ syringe. If the perforation was over medium sized, ciprofloxacin-dexamethasone impregnated gelfoam was packed into the middle ear via the perforation.

After completion of middle ear packing, fat was inserted into the perforation with a alligator forcep and pick. The stability of the fat graft was identified using fine suction tip. If the perforation size is large than $50 \%$ after deepithelization of the perforation margin, the compressed fat was used after middle ear packing. After taken fat as large as possible from the earlobe, the fat was compressed by House gelfoam press (Fig. 2). The compressed fat graft was placed in the perforation by transtympanic underlay myringoplasty technique and supported by fibrin glue on the fat graft and external bony canal. Although fat graft was compressed, the compressed fat is more thicker than fascia graft, thick compressed fat tissue is better for adapting to the vascular bed than a thin fascia graft [24,25].

It is important to ensure that even distributed fat tissue contacts to the all perforation margin using very fine suction tip. The patients were instructed to avoid water in the operated ear for three weeks. The patients were followed up 3 weeks postoperatively. After watchful observation using otoendoscope, patients were instructed to use ototopical antibiotics drops (ciprofloxacin) which resolves the dried gelfoam and dried fibrin glue over the fat graft and stabilized the tympanization. The $1^{\text {st }}$ follow-up was performed 3 weeks after surgery, patient was instructed to put the otic drop (cifloxacin) twicely per day for the resorbing the glue remnant over the fat graft. The fat graft could be evaluated by endoscopy 6 weeks after surgery, majority of the patients showed the intact neodrum with neovascularization in the surface. If the fat graft shows incomplete epithelization, the additional self-putting otic drop is recommended. The majority of the patients shows complete epithelization of the neodrum within 6-8 weeks postsurgery. The postoperative pure-tone audiogram was performed.

\section{Statistical analysis}

Statistical analysis was performed using Prism software (v 8.8; GraphPad). The preoperative and postoperative hearing assessment was performed by $t$ test. The data were considered statistically significant when the $p$ value was less than 0.05 .

\section{Results}

Two hundred nine patients were included in this study. The mean age of the patients in this study was 58.35 (range 3-86). 60.2 (range, 13-86) in female and 56.5 (range 3-85) in male. The success rate was divided by age. The mean successful TM closure rate was $88.0 \%$. The success rate by different age generation was not significant difference. The success rate was 93.3\% (10-20s), 82.4\% (30s), 96.2\% (40s), 81.6\% (50s), 86.1\% (60s), 87.2\% (70s), 95.5\% (80s) (Fig. 3). Although seventies or eighties, success rate was high in eighties $95.5 \%$.

The successful closure rate by different size of perforation was as follows; $88 \%$ in pinhole size (n=38/43), $90.9 \%$ in grade I ( $n=110 / 121), 78.8 \%$ in grade II ( $n=26 / 33$, follow-up loss 1$), 81.8 \%$ in grade III ( $n=9 / 11)$. The size of the perforation grade showed no significant difference (Fig. 4). The success rate according to the perforation site was divided as anterior and posterior less than grade I. The success rate of the anterior perforation was $88.5 \%(n=131 / 148)$ and $85.2 \%(n=52 / 61)$. The perforation site by anterior or posterior was not significant. The hearing change of pure conductive hearing loss was evaluated at 6 months postoperatively. The preoperative mean threshold of AC, BC and ABG were 55.71, 12.98 and $42.73 \mathrm{~dB}$ respectively. The postoperative mean threshold of AC, BC and ABG were 23.67, 12.98 and 10.69 dB (Fig. 5). The change of preoperative and postoperative hearing showed statistically significant $(\mathrm{AC}$ threshold: $p=0.0001, \mathrm{BC}$ threshold: $p=0.0002)$. 


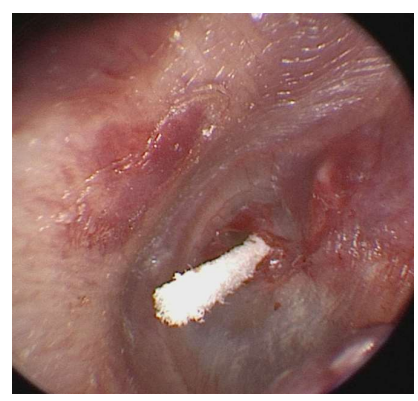

Figure 1. Toothstick-like gelfoam was inserted into the small perforation for middle ear packing.

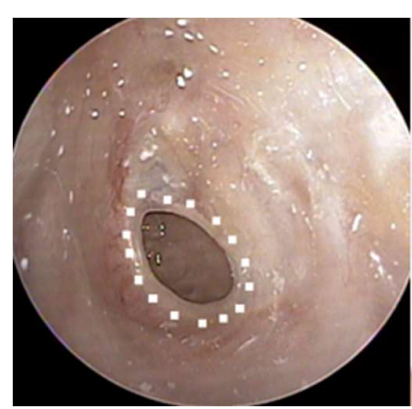

Figure 2A

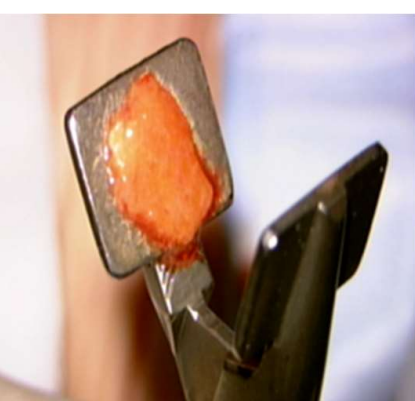

B

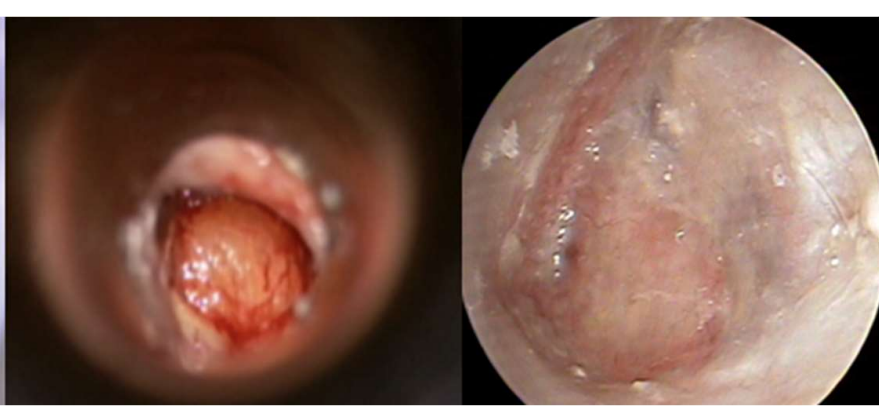

C
D.

After trimming of perforation, the perforaton size can be large perforation (A). The compression makes the fat as flat graft (B). The compressed fat was placed by transtympanic underlay technique after middle ear packing using gelfoam. The postoperative endoscopy shows intact neodrum at 6 weeks postsurgery.

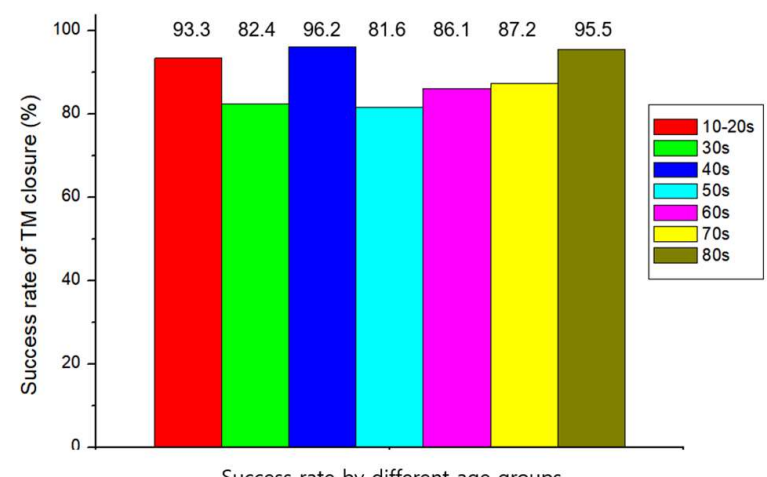

Figure 3. The success rate by different age generation was not significant difference. 


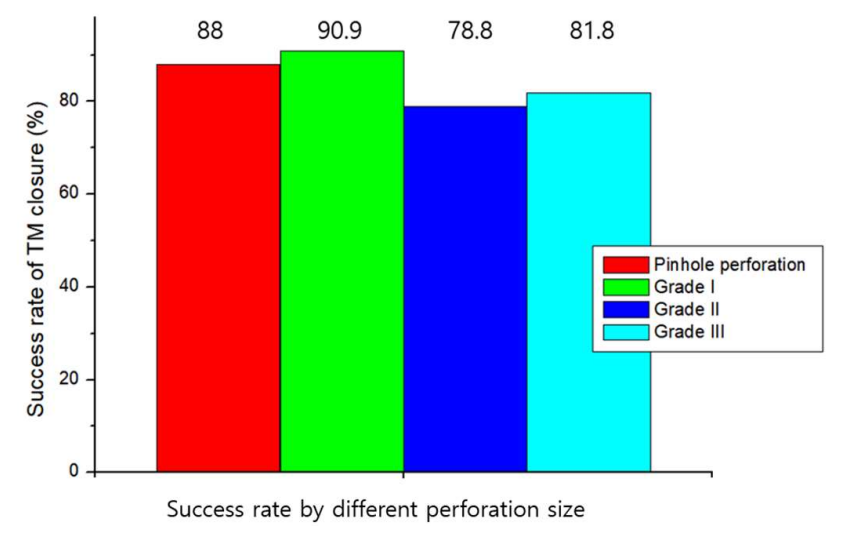

Figure 4. The size of the perforation grade showed no significant difference.

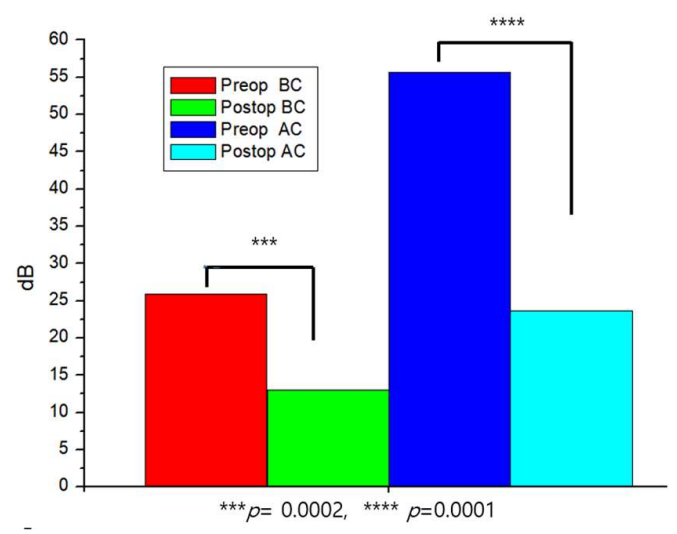

Figure 5. The change of preoperative and postoperative hearing showed statistically significant (AC threshold: $p=$ 0.0001, BC threshold: $p=0.0002$ ).

\section{Discussion}

The rate of nature closing of the chronic TMP is very rare. The TMP margin is generally covered by squamous epithelium and ingrowth to the medial layer. Tympanoplasty requires middle ear exploration by tympanomeatal flap elevation. Compared to conventional tympanoplasty, fat myringoplasty does not require this tympanotomy procedure.

Fat myringoplasty with hyaluronic acid (Epidisc) was tried and introduced that hyaluronic acid resulted in higher success rate compared to simple fat myringoplasty $[14,17]$. However, the success rate of simple fat myringoplasty group was $69.9 \%$ in studies that compared it with hyaluronic acid fat myringoplasty. This success rate of simple fat myringoplasty group quite different from other's report. However, the success rate of simple fat myringoplasty group was $69.9 \%$ in studies that compared it with hyaluronic acid fat myringoplasty. This success rate of simple fat myringoplasty group quite different from other's report. Our results of graft success rate was $88.0 \%$. And we compared to hyaluronic acid fat graft group $84-90.1 \%$ by Saliba [17], there was no significance between two groups. The success rate according to the size of the perforation was controversial. Deddens et al [6], and Konstantinidis et al reported that TM perforation size is a critical factor for success rate and they recommend that fat myringoplasty should be applied when the TM perforation size is less than 30\% of TM. Li et al [7], Kim et al [5], Gun et al [26], and Konstantinidis et al [2] showed poor success rate in large perforation but Knutsson et al [10] reported higher success rate in large perforation than small perforation. In this study, it was not proportional to the size of the TM perforation. The compressed fat was applied in the large perforation and old aged patients. They showed good success rate. Saliba [17] reported that he designed the harvested fat as a hourglass shaped plug and inserted to the peforation site. He also used gelfoam packing in the middle ear when the perforation size is over than medium size. Compared to hourglass shaped plug, our compressed fat is easy to insert to the perforation. And the physiologically, hourglass shaped fat plug in larger perforation which may 
obliterate the middle ear and it can induce poor acoustic property. However, our compressed fat graft does not obliterate the middle ear cavity because its shape is flat and we performed middle ear packing using ciprofloxacin/dexamethasone soaked gelfoam which support the fat graft. Sakagami[25] and Yuasa [25,27] reported subcutaneous connective tissue much better than temporalis muscle fascia in simple underlay myringoplasty because thick subcutaneous connective tissue is better at adapting. The compressed fat is more thicker than fascia, therefore it is easy to adapt.

In this study, we performed middle ear packing using ciprofloxacin/dexamethasone soaked gelfoam.

It is closely associated with fibrosis and adhesion formation in the middle ear as well as airway mucosa. Fibroblasts and $\alpha$-SMA (in response to profibrotic agents such as transforming growth factor-beta, TGF- $\beta$ ) play a important role in fibrosis formation [28]. Dexamethasone reduced expression of alpha-smooth muscle actin and the short isoform of myosin light chain kinase in the airway mucosa [29].

Previous our experiment, we performed an in vitro and in vivo evaluation of the antifibrotic effects of a dexamethasone and alginate coating on silicone sheet. The dexamethasone-coated silicone sheets effectively limited in vitro fibroblast attachment and proliferation due to the controlled release of dexamethasone. And dexamethasone showed antifibrotic effect with inhibition of the expression of alpha SMA in the guinea pig mucosa [19]. The middle ear packing supports the graft for stability and prevention of retraction. The best position of the graft is placed between perforation of the tympanic membrane and gelfoam bed in the middle ear with autograft such as fat or perichondrium. The middle ear packing using dexamethasone soaked gelfoam showed antifibrotic effect [30].

The fibrin glue packing over the compressed fat graft by simple underlay technique is helpful in better hearing ear or geriatric patient because it is simple process without elevation of tympanomeatal flap.

Actually, Sakagami et al [25] performed simple underlay myringoplasty without middle ear packing, but the middle ear packing using dexamethasone soaked gelfoam supports the compressed fat graft in larg]perforation. By Sakagami et al [25] Yuasa et al [27], the postoperative re-perforation rate by simple underlay myringoplasty without middle ear packing was higher than that for conventional methods. The success rate was 76-77\%. Actually their success rate was lower than our success rate. We think middle ear packing enhances the success rate.

In this study, audiologic outcome in pure conductive hearing loss patients, the AC and BC threshold were improved after operation. The BC threshold can be increased in chronic otitis media due to both mechanical and chemical factors and $\mathrm{BC}$ threshold can be improved by tympanoplasty or tympanomastoidectomy not only close air bone gap but also improve BC threshold [31].

In our results, there was no significant difference of the closure rate in each size of perforation. In case of large perforation, the middle ear packing and fibrin glue over the compressed fat graft plays a role of stabilization of compressed fat graft on success rate of TMP closure.

\section{Conclusion}

From our results, the simple trimming of the perforation edge and middle ear packing with external ear canal packing using fibrin glue induced the stable adhesion between the remnant TM and the fat graft. 
Author Contributions: For research articles with several authors, a short paragraph specifying their individual contributions must be provided. The following statements should be used “Conceptualization, CHJ.; methodology, CHJ.; formal analysis, JK.; investigation, JK.; data curation, JK.; writing - original draft preparation, JK.; writing - review and editing, CHJ.; funding acquisition, CHJ All authors have read and agreed to the published version of the manuscript." Please turn to the CRediT taxonomy for the term explanation. Authorship must be limited to those who have contributed substantially to the work reported.

Funding: This work was supported by grants from the Basic Science Research Program through the National Research Foundation of Korea (NRF) funded by the Ministry of Education, Science and Technology (NRF-2018-RID-1A1B07048074), National Research Foundation of Korea (NRF) funded by the Ministry of Science and ICT for Bioinspired Innovation Technology Development Project (NRF-2018M3C1B7021997) and the Korea Medical Device Development Fund grant funded by the Korea government (the Ministry of Science and ICT, the Ministry of Trade, Industry and Energy, the Ministry of Health \& Welfare, Republic of Korea, the Ministry of Food and Drug Safety) (Project Number: 202013C05).

Institutional Review Board Statement: The study was conducted according to the guidelines of the Declaration of Helsinki, and approved by the Institutional Review Board of Chonnam National University Hospital (approval No. CNUH-2020-153).

Conflicts of Interest: The authors declare no conflict of interest.

\section{References}

1. Ringenberg, J.C. Closure of tympanic membrane perforations by the use of fat. Laryngoscope 1978, 88, 982-993, doi:10.1288/00005537-197806000-00010.

2. Konstantinidis, I.; Malliari, H.; Tsakiropoulou, E.; Constantinidis, J. Fat myringoplasty outcome analysis with otoendoscopy: who is the suitable patient? Otol Neurotol 2013, 34, 95-99, doi:10.1097/MAO.0b013e318278c1e3.

3. Acar, M.; Yazıc1, D.; San, T.; Muluk, N.B.; Cingi, C. Fat-plug myringoplasty of ear lobule vs abdominal donor sites. Eur Arch Otorhinolaryngol 2015, 272, 861-866, doi:10.1007/s00405-014-2890-0.

4. Diaz, A.R.; Reina, C.O.; Plaza, G.; Posadas, E.R.; Arevalo, F.V.; Iriarte, M.T.G. Long-Term Follow-Up After Fat Graft Myringoplasty: Do Size and Location Matter? Ear Nose Throat J 2021, 100, 229s-234s, doi:10.1177/0145561320973555.

5. Kim, D.K.; Park, S.N.; Yeo, S.W.; Kim, E.H.; Kim, J.E.; Kim, B.Y.; Kim, M.J.; Park, K.H. Clinical efficacy of fat-graft myringoplasty for perforations of different sizes and locations. Acta Otolaryngol 2011, 131, 22-26, doi:10.3109/00016489.2010.499881.

6. Deddens, A.E.; Muntz, H.R.; Lusk, R.P. Adipose myringoplasty in children. Laryngoscope 1993, 103, 216-219, doi:10.1002/lary.5541030217.

7. Li, P.; Yang, Q.T.; Li, Y.Q.; Liu, W.; Wang, T.; Li, Y. The selection and strategy in otoendoscopic myringoplasty with autogenous adipose tissue. Indian J Otolaryngol Head Neck Surg 2010, 62, 25-28, doi:10.1007/s12070-010-0010-z.

8. Koc, S.; Akyuz, S.; Gurbuzler, L.; Aksakal, C. Fat graft myringoplasty with the newly developed surgical technique for chronic tympanic membrane perforation. Eur Arch Otorhinolaryngol 2013, 270, 1629-1633, doi:10.1007/s00405-012-2040-5.

9. Gun, T.; Sozen, T.; Boztepe, O.F.; Gur, O.E.; Muluk, N.B.; Cingi, C. Influence of size and site of perforation on fat graft myringoplasty. Auris Nasus Larynx 2014, 41, 507-512, doi:10.1016/j.anl.2014.08.004.

10. Knutsson, J.; Kahlin, A.; von Unge, M. Clinical and audiological short-term and long-term outcomes of fat graft myringoplasty. Acta Otolaryngol 2017, 137, 940-944, doi:10.1080/00016489.2017.1326063.

11. Saliba, I.; Froehlich, P. Hyaluronic acid fat graft myringoplasty: an office-based technique adapted to children. Archives of otolaryngology--head \& neck surgery 2011, 137, 1203-1209, doi:10.1001/archoto.2011.188.

12. Saliba, I. Hyaluronic acid fat graft myringoplasty: how we do it. Clinical otolaryngology : official journal of ENT-UK ; official journal of Netherlands Society for Oto-Rhino-Laryngology \& Cervico-Facial Surgery 2008, 33, 610-614, doi:10.1111/j.17494486.2008.01823.x.

13. Saliba, I. In response to Growth factors expression in hyaluronic acid fat graft myringoplasty. Laryngoscope 2017, 127, E252, doi:10.1002/lary.26630.

14. Saliba, I.; Alzahrani, M.; Zhu, T.; Chemtob, S. Growth factors expression in hyaluronic acid fat graft myringoplasty. Laryngoscope 2014, 124, E224-230, doi:10.1002/lary.24468.

15. Saliba, I.; Knapik, M.; Froehlich, P.; Abela, A. Advantages of hyaluronic acid fat graft myringoplasty over fat graft myringoplasty. Arch Otolaryngol Head Neck Surg 2012, 138, 950-955, doi:10.1001/archotol.2013.210.

16. Saliba, I.; Woods, O. Hyaluronic acid fat graft myringoplasty: a minimally invasive technique. Laryngoscope 2011, 121, 375380, doi:10.1002/lary.21365.

17. Saliba, I. Hyaluronic acid fat graft myringoplasty: how we do it. Clinical Otolaryngology 2008, 33, 610-614.

18. Merchant, S.N., Nadol JB. Schuknecht's Pathology of the Ear, 3rd ed.; Merchant, S.N., Nadol JB, Ed.; People's medical publishing house: 2010.

19. Jang, C.H.; Ahn, S.H.; Kim, G.H. Antifibrotic effect of dexamethasone/alginate-coated silicone sheet in the abraded middle ear mucosa. Int J Biol Macromol 2016, 93, 1612-1619, doi:10.1016/j.ijbiomac.2016.04.033. 
20. Jang, C.H.; Cho, Y.B.; Choi, C.H.; Lee, J.S.; Kang, S.I. Effect of anti-adhesion barrier solution containing ciprofloxacinhydrocortisone on abraded mucosa with otitis media. International journal of pediatric otorhinolaryngology 2013, 77, 19-24.

21. Lehner, E.; Liebau, A.; Syrowatka, F.; Knolle, W.; Plontke, S.K.; Mäder, K. Novel biodegradable Round Window Disks for inner ear delivery of dexamethasone. Int J Pharm 2021, 594, 120180, doi:10.1016/j.ijpharm.2020.120180.

22. Scheper, V.; Hessler, R.; Hütten, M.; Wilk, M.; Jolly, C.; Lenarz, T.; Paasche, G. Local inner ear application of dexamethasone in cochlear implant models is safe for auditory neurons and increases the neuroprotective effect of chronic electrical stimulation. PLoS One 2017, 12, e0183820, doi:10.1371/journal.pone.0183820.

23. Eom, T.H.; Lim, H.R.; Jeong, S.H.; Park, K.S.; Jang, C.H. Hearing Results Following Type 1 Tympanoplasty in Elderly Patients. In Vivo 2020, 34, 1395-1398, doi:10.21873/invivo.11919.

24. Sakagami, M.; Mishiro, Y.; Tsuzuki, K.; Seo, T.; Sone, M. Bilateral same day surgery for bilateral perforated chronic otitis media. Auris Nasus Larynx 2000, 27, 35-38, doi:10.1016/s0385-8146(99)00043-7.

25. Sakagami, M.; Yuasa, R.; Yuasa, Y. Simple underlay myringoplasty. J Laryngol Otol 2007, 121, 840-844, doi:10.1017/s0022215106005561.

26. Gün, T.; Boztepe, O.F.; Atan, D.; İkincioğulları, A.; Dere, H. Comparison of Hyaluronic Acid Fat Graft Myringoplasty, Fat Graft Myringoplasty and Temporal Fascia Techniques for the Closure of Different Sizes and Sites of Tympanic Membrane Perforations. J Int Adv Otol 2016, 12, 137-141, doi:10.5152/iao.2016.1938.

27. Yuasa, Y.; Yuasa, R. Postoperative results of simple underlay myringoplasty in better hearing ears. Acta oto-laryngologica 2008, 128, 139-143.

28. Sousa, A.M.; Liu, T.; Guevara, O.; Stevens, J.; Fanburg, B.L.; Gaestel, M.; Toksoz, D.; Kayyali, U.S. Smooth muscle $\alpha$-actin expression and myofibroblast differentiation by TGF $\beta$ are dependent upon MK2. Journal of cellular biochemistry 2007, 100, 1581-1592.

29. Goldsmith, A.M.; Hershenson, M.B.; Wolbert, M.P.; Bentley, J.K. Regulation of airway smooth muscle alpha-actin expression by glucocorticoids. Am J Physiol Lung Cell Mol Physiol 2007, 292, L99-1106, doi:10.1152/ajplung.00269.2006.

30. Deniz, B.; Oguzhan, K.R.; Erdem, O.; Hasan, S.; Fuat, Y.Y.; Muge, O. The effects of different packing materials on healing and hearing after trauma to middle ear mucosa, an experimental study in rats. Am J Otolaryngol 2019, 40, 347-352, doi:10.1016/j.amjoto.2019.01.006.

31. Vartiainen, E.; Karjalainen, S. Factors influencing sensorineural hearing loss in chronic otitis media. Am J Otolaryngol 1987, 8, 13-15, doi:10.1016/s0196-0709(87)80013-3. 